so prevalent in our society and regrettably in our own profession.

As a recovering alcoholic, with only just over one year's sobriety, I feel that I am in a position to stress the great help I have received from Alcoholics Anonymous. Only by regular attendances and by following their programme have I been able to control this disease up to today. Anyone who feels that they may have a drink problem are welcomed at their loca meetings, and may come and go as they please and surname anonymity is a top priority. The nearest meeting for your patient can be had from the Central Office in London by phoning 01-352 9779

Doctors and dentists with a drink/drug problem may also find help and encouragement by attending the monthly meetings of the British Doctors' and Dentists' Group. This is an independent group that has regular meetings in Northern Ireland, Glasgow, Durham, Manchester, Bristol, Petersfield (Hants), and London. Many recovering doctors and dentists attend these meetings as well as Alcoholics Anonymous meetings. The Doctors' Group may be contacted through the Medical Council on Alcoholism, telephone 012354182 . All inquiries will be dealt with in the strictest confidence.

A doctor member of Alcoholics Anonymous

\section{Assessment of iron stores in inflammation by assay of serum ferritin concentrations}

SIR,-Dr D R Blake and others studied serum ferritin concentrations in inflammatory synovitis (31 October, p 1147). They did not exactly demonstrate a correlation between ferritinaemia and iron stores, since no direct measurement was performed in their patients. Rather they pointed out that a serum ferritin concentration below $55 \mu \mathrm{g} / 1$ indicates iron deficiency complicating chronic inflammation. From a practical point of view, it is very useful to have this upper limit defined, as the classica value of transferrin saturation below $0 \cdot 16^{1}$ is not very helpful for detecting iron deficiency in chronic disorders. These findings have been verified in another kind of inflammatory disease-namely, chronic pulmonary tuberculosis.

We have studied 28 adult patients ( 24 women and four men). Our findings were reported to the $18 \mathrm{th}$ congress of the International Society of Haematology, Montreal, in August 1980. Iron stores were evaluated directly, marrow haemosiderin being graded from 0 to $6+$ (low, 1-2; normal, 3-4; increased, 5-6 $)^{2}$ and the percentage of marrow sideroblasts being determined. At the same time serum ferritin concentration was determined by a commercially available radioimmunoassay (FerK-Kit, CIS, France), and transferrin saturation was measured by a standard method (BD-Merieux, France). A positive correlation was observed between the ferritin concentration and haemosiderin score (log ferritin value $v$ haemosiderin score: $r=0.5108, p<0.01$, but not between the transferrin saturation and haemosiderin score $(r=0.0540)$.

The ferritin concentration was below $55 \mu \mathrm{g} / \mathrm{l}$ (4-44) in a group of five women, with an average haemosiderin score below $1(0,0,1,1,2)$ and no marrow sideroblasts. The ferritin concentration exceeded $55 \mu \mathrm{g} / 1$ in the 23 others, with a mean haemosiderin score value of 3.5 (low, normal, and increased in 4, 12 and 7 cases respectively), no marrow sideroblasts in eight cases, and $1-14 \%$ in 15. The transferrin saturation values were below 0.16 in 19 patients, whose mean haemosiderin score was 3.0 (low, normal, and increased in eight, six, and five cases respectively).

Thus, of the indirect methods for evaluating iron stores, determining the serum ferritin concentration is better correlated with a direct method than is determination of the transferrin saturation in patients suffering from chronic diseases (arthritis, tuberculosis, regional enteritis, ${ }^{3}$ or even sickle-cell anaemia ${ }^{4}$ ) complicated by iron deficiency; and the latter condition can be safely inferred from a serum ferritin concentration below $55 \mu \mathrm{g} / 1$.

K BELDJORD

K BENLATRACHE

R Colonna

Haematology Clinic,

Centre P et $M$ Curie,

Hopital Mustapha,

1 Bainton DF, Finch CA. Am f Med 1964 ;37:62-70. Baumgartner-Staubli R, Back EA. Acta Haemato 1977;57:24-31

Thomson ABR, Brust R, Ali AM, Mant MJ, Valberg LS. Am f Dig Dis 1978;23:705-9.

$1981 ; 58: 563-8$.

Fake!

SIR,-“Fake," the fascinating article by Dr $T$ J Hamblin (19-26 December, p 1671) prompts me to recall a particularly audacious example of scientific faking of half a century ago, in which I was very indirectly involved.

In 1930 the Lancet published an article of eight full pages entitled "The aetiology of disseminated sclerosis."' The author, Kathleen Chevassut, MSc claimed to have isolated a specific pathogen from the cerebrospinal fluid of 176 of 189 patients with multiple sclerosis but never from 269 patients with other organic nervous diseases, almost burying this startling discovery beneath a mass of technical details. For this work she had been assisted by grants from the Medical Research Council (MRC) the University of London, and the John Burford Carlill Endowment. Sir James Purves-Stewart, physician to Westminster Hospital, who had requested her to undertake this research, had made her a personal gift of a "special microscope and other apparatus," and had provided the clinical material. According to Miss Chevassut, her organism bore a "striking resemblance to the organism which is regarded as the causative agent of bovine pleuro-pneumonia."

Chevassut's article was immediately followed by a five-page report by Purves-Stewart called "A specific vaccine treatment in disseminated sclerosis." $\mathrm{He}$ had requested Chevassut to prepare from her cultures of the organism-which they had decided to name Spherula insularis-autogenous vaccines, which were administered intravenously to 128 patients, with "suggestive" results.

Attempts by the MRC to make an independent appraisal of Chevassut's claims were thwarted by her evasiveness, but early in 1931 the Halley Stewart Trust decided to endow an institute at Hampstead to enable her to pursue her work under the best conditions. ${ }^{3}$ This consisted of nursing staff and a laboratory assistant and accommodation for 12 inpatients, Chevassut also receiving a personal grant. The philanthropist Sir Halley Stewart persuaded one of his sons-Dr B Halley Stewart, who had retired-to accept the position of medical superintendent of the institute, but he left after a few weeks because "Miss Chevassut resented my presence there."

Over four months later Dr Halley Stewart returned to the institute in order to establish "a system of controls of all the lumbar punctures." According to him, Chevassut's strategems to meet this new challenge included replacing culture tubes by "dummy tubes," turning the incubator up to $50^{\circ} \mathrm{C}$, and making new slides of her Spherula insularis from "four or five culture tubes which she carried about with her in the pocket of her white coat." In the meantime, S R Douglas, FRS, of the MRC, reported that four "positives" from Chevassut showed "a pure culture of the virus of bovine pleuropneumonia."

By then Chevassut was totally discredited, and Purves-Stewart publicly "dissociated" himself from her-but not from Spherula insularis. He continued to find the organism not only in the cerebrospinal fluid of multiple sclerosis patients but also in that of patients with other organic nervous diseases, and in pus-containing fluids from the pleura, the peritoneum, and syphilitic chancres. Far from being discouraged by the apparent non-specificity of "Spherula," he prepared from the cerebrospinal fluid of 101 multiple sclerosis patients "autogenous vaccines," the administration of which allegedly produced arrest or clinical improvement in most of them.

In 1933 I became a house physician to the Metropolitan Hospital, Kingsland Road, London, and found that Chevassut was continuing her operations there, clad in a white coat and brewing her "autogenous vaccines" under the patronage of the neurologist C Worster-Drought, FRCP, who was also physician to the West End Hospital for Nervous Diseases. His private patients came from many parts of the country to be admitted to the Metropolitan Hospital for a few days for lumbar puncture, culture of the "pathogen," and administration of the "vaccine." On many occasions I had the distasteful duty of participating in this exploitation of the sick by performing the lumbar punctures on them.

Geneva, Switzerland

NORMAN HOWARD-JONES

\section{Chevassut K. Lancet 1930; i:552-60.}

Purves-Stewart J. Lancet $1930 ; \mathrm{i}: 560-4$.

SIR,-Dr T J Hamblin's enlightening article (19-26 December, $p$ 1671) on the dark corners of science speaks of Burt as, firstly, fabricating his evidence and, secondly, fixing his facts. The evidence would seem to be that his earlier data were reliable, but their numerical interpretation became "fixed" beyond the likely vagaries of chance despite further, but poorly documented, data.

As with Mendel and, in a similar context, Newton, there is no reason to doubt the magnitude of the numerical value, which seems consistent with the mechanism and with other studies. It is hardly Burt who should be blamed for the widespread confusion between heritability and ineducability.

J H EDWARDS

Genetics Laboratory,

University Department of Biochemistry,
Oxford OX1 3QU

Gonadotrophin and the human secondary sex ratio

SIR,-I have suggested ${ }^{12}$ that the sex ratio (proportion of boys) of infants born after the induction of ovulation is low. Dr Ina Cholst and others (7 November $p$ 1264) report the births of 79 boys and 73 girls after the induction of ovulation. They correctly write : “... our data do not support a recent report of an alteration in the sex ratio in infants conceived after the induction of ovulation." It seems nevertheless fair to add that their data do not powerfully impugn the hypothesis either: the sex ratio of their sample is not significantly different from that of the sex ratio of the 257 boys and 333 girls in the study already referred to.

The accompanying table gives the sexes of the infants in further samples which have be- 
Sexes of infants born after induction of ovulation

\begin{tabular}{lrr}
\hline Source* & Girls & Boys \\
\hline F J Beernink (private communication) & 4 & 12 \\
J B Brown (private communication) & 104 & 104 \\
S Byrne (private communication) & 40 & 41 \\
R N Turksoy et al & 2 & 5 \\
R S Neuwirth et al & 1 & 3 \\
G C Liggins and H K Ibertson & 1 & 4 \\
R A Aiken & 2 & 4 \\
M C Botha et al & 4 & 0 \\
R D Atlay and G W Pennington & 2 & 1 \\
N Eckstein and M P David & 4 & 2 \\
W J Garrett et al & 5 & 4 \\
W Mobius and W Carol & 4 & 4 \\
M Granat & 4 & 0 \\
Giovannucci Uzielli et al & 4 & 2 \\
L Varga et al al Ouist & 2 & 3 \\
J Cohen and C Quist & 0 & 2 \\
J C Monnier et al & 1 & 4 \\
T A Walker and W F Baden & 2 & 0 \\
\hline Total & 186 & 195 \\
\hline
\end{tabular}

*Full references are available on request.

come known to me since that publication. There is no evidence for heterogeneity among these various sets of data so it seems reasonable to pool them. This yields 522 boys and 601 girls. When tested against an expected sex ratio of 514 , this sample gives a chi-squared value of $10.86, p \bumpeq 0.001$. So taken together the data still powerfully support the hypothesis.

As the techniques of hormonal monitoring improve, the amount of administered hormone will presumably decrease. So if the hypothesis is correct, the departures from expected sex ratios among children born following induced ovulation should become progressively less. Working with mice, Elbling ${ }^{3}$ has given strong evidence that under some circumstances the administration of gonadotrophin is associated with a subsequent low sex ratio in the pups. Smith and Chrisman 5 failed to reproduce this result; but such is the strength of Elbling's evidence that one might infer not that the hypothesis may be safely dismissed but that a phenomenon does exist which is incompletely understood.

It would be useful if other workers would repeat Elbling's experiment. I should also be glad to be contacted by anyone with unpublished data on the sex of infants following induced ovulation.

I am grateful to Dr F J Beernink (of Berkeley, California), Professor J B Brown (University of Melbourne), and Sister S Byrne (of the Women's Hospital, Surrey Hills, New South Wales) for data in tabulated form.

WILLIAM H JAMES

University College London

MRC Mammalian Development Unit,
London NW $12 \mathrm{HE}$

1 James WH. Br Med $\mathcal{F}$ 1980;281:711-2.

2 James WH. Lancet 1980;1:1124-6. Elbling L. Proc Soc Exp Biol Med 1975;149:376-9.
smith CM, Chrisman CL. Nature 1975;253:631-2.

\section{Reduction of free testosterone by}

\section{antiepileptic drugs}

SIR,-This tidy research work by Dr Jila Dana-Haeri and others (9 January, p 85) prompted me to contemplate the role of sex hormones in epileptics with acne vulgaris. Typical adolescent acne is found in epileptic boys under treatment long before puberty, and at one time I even thought that phenytoin had a virilising effect as epileptic women taking it had hirsutism. Adult epileptics are prone to very severe acne, which is resistant to local applications that are usually helpful; and this might imply a more severe sex hormone imbalance in epileptics.
The endocrine factors causing acne are complex, but androgens seem to play a dominant role. Acne is not seen in the castrated, and antiandrogens produce improvement albeit with loss of libido. Furthermore oral contraceptives, provided that they contain a non-androgenic progestogen, afford beneficial results in both sexes. It would seem therefore that acne results from an imbalance of sex hormones with a relative preponderance of androgens; and epileptics under treatment tend to suffer from early, severe, and stubborn acne.

Bearing in mind these clinical observations, I remain somewhat bewildered by the findings of Dr Dana-Haeri and others, who found a reduced concentration of plasma free testosterone in epileptics under treatment with antiepileptic drugs.

IAN MARTIN-SCOTT

Chorleywood, Herts WD3 4BW

\section{Squash ball to eye ball}

SIR,-May I add to the correspondence concerning squash ball eye injuries with a comment on injury to the eye from a squash racket?

Fourteen years ago I was hit by a squash racket in the left eye, which resulted in severe loss of vision due to a retinal haemorrhage. Not wishing to give up playing squash I had a metal mask constructed (figure). It does not

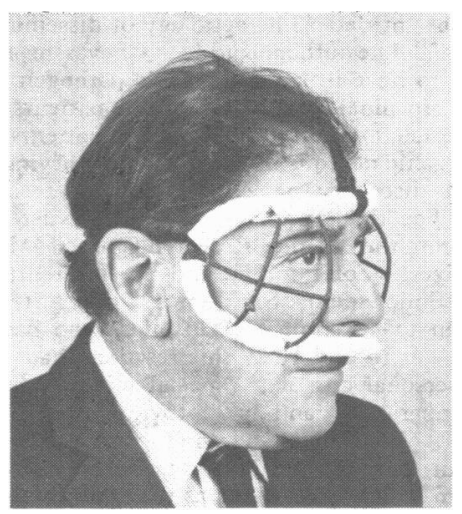

Protective mask for a squash player.

obstruct the vision in any way and will prevent both a squash ball and a squash racket from damaging the eye. If anyone would be interested in further information I would be happy to supply it.

E D KERR

Barn Cottage,

Cryers Hill Lane,

Widmer End

High Wycombe, Bucks

\section{Smoking, IgE, and occupational allergy}

SIR,-Dr O Zetterström and his colleagues (7 November, $p$ 1215) report that raised serum IgE concentrations and increased risk of occupational allergy may represent another hazard of smokers. Our recent work (reported to the 15th Italian National Congress of Allergology and Clinical Immunology, 1981) seems in part to confirm this finding and to provide additional stimulus for further research on this new subject.

In a survey carried out in collaboration with Professor G Berlinguer (Institute of Physiology and
Occupational Hygiene, University of Rome) to assess damage induced by an occupational exposure to cosmetics, serum IgE levels were measured in 320 hairdressers and 201 controls by PRIST (Phadebas IgE, Pharmacia, Uppsala). Lung function tests were also carried out. IgE concentration in the serum was related to the age of the subjects, the presence of current or previous allergic symptoms, occupational exposure to cosmetics, and smoking habits (subjects smoking more than five cigarettes a day being considered as smokers) Statistical analysis was performed on the natural logarithm (In) of the IgE values by Professor L Piccinato (Institute of Probability, Faculty of Statistics, University of Rome) by regression analysis.

While total serum IgE was not influenced by the age of the subjects or exposure to cosmetics, a history of allergy and, more interestingly, smoking habits were significantly related to high $\operatorname{IgE}$ values $(p<0.05)$. No significant additive effect was observed between the two variables. Mean levels of serum IgE in smokers and non-smokers are shown in the table.

Serum IgE levels in smokers and non-smokers

\begin{tabular}{lcc}
\hline & No & In IgE (mean \pm SD) \\
\hline Smokers & 222 & $4 \cdot 12 \pm 1 \cdot 57$ \\
Non-smokers & 283 & $3 \cdot 79 \pm 1.33$ \\
< 0.05. & & \\
\hline
\end{tabular}

Professor Zetterström and his colleagues report that raised $\operatorname{IgE}$ values are due to an increased frequency of sensitisation in smokers, possibly related to the damage inflicted by smoking on the bronchial mucosa. This was not manifest in our data, the frequency of allergy being in fact similar in smokers and non-smokers. On the contrary, the statistical analysis we performed showed that the effect of smoking on IgE levels was independent of the occurrence of clinical sensitisation. Moreover, even when allergic subjects were excluded from the sample, the 133 smokers still had higher IgE concentrations than the 182 non-smokers $(4.09$ 土 $1.56 v 3.69+1.28 \ln \mathrm{IgE} ; \mathrm{p}<0.05)$. This aspect also emerged from the study of $\mathrm{Dr}$ Zetterström and his co-workers, the difference in IgE serum levels between smokers and non-smokers reaching an even higher statistical significance when only non-atopic subjects were considered.

Smoking habits may certainly influence the target organ of a clinical hypersensitivity (that is, the bronchi), thus possibly explaining the higher frequency of allergic asthma in smokers. ${ }^{1}$ However, on the basis of our findings, we would like to suggest the possibility of a more direct effect of smoking on IgE production, apart from that of an increased frequency of sensitisation to smoke or occupational or common allergens. Daniele and coworkers reported alterations in smokers in the morphology and function of alveolar macrophages and a reduced proliferative lymphocyte response to phytohaemagglutinin and concanavallin A. ${ }^{2}$ This could imply a toxic effect of smoking on various immune cells, including those involved in IgE synthesis. Smoking has in fact been shown to cause significant changes in other immunoglobulin classes. $^{3}$

It is commonly reported that the distribution of serum IgE values in healthy subjects is not Gaussian. In our opinion this implies an inadequacy of the criteria used for selecting normal subjects, as yet unrecognised environmental or pathological factors being responsible for the heterogeneity of the so-called "normal" population. The effect of smoking on IgE suggests that smokers should be excluded from all studies of normal IgE levels. Our study of a large number of subjects provides prompt confirmation of the reports of Gerrard and coworkers ${ }^{4}$ and of Professor Zetterström and his colleagues that healthy subjects should bear in mind the risk that smoking, among other things, could increase their IgE levels. The 